\title{
The role of extramaze cues in spontaneous alternation in a plus-maze
}

\author{
ROBERT C. LENNARTZ \\ Indiana University Southeast, New Albany, Indiana
}

\begin{abstract}
Performance on various spatial tasks is superior in the presence of extramaze cues that provide guidance in locating the goal. This study was an investigation of the effect of such cues on nonrewarded, continuous spontaneous alternation in a plus-maze, a spatial task with no explicit reinforcement. Rats were tested in five daily sessions. Alternation scores, which reflect how frequently the least recently visited arms are chosen, were calculated for each day. On the first day only, rats in the stimulus-rich condition, with cues added to the curtain that surrounded the maze, alternated more than did rats in the stimulus-poor condition. Over days, the stimulus-rich group showed a significant downward trend in alternation, whereas the stimulus-poor group showed an upward trend, but all the groups always alternated at levels greater than chance. Thus, although performance on this task can be enhanced by the presence of extramaze cues, other types of information support alternation.
\end{abstract}

Spontaneous alternation is a spatial task in which there is no obvious reward for making the correct choice. It is has often been studied using a T-maze, with the bottom of the "T" as the start point; rats approaching the choice point from the start arm tend to choose the arm not visited on the previous trial (Dennis, 1935; Tolman, 1925). Since the initial reports of the phenomenon, various aspects of spontaneous alternation behavior in a T-maze have been extensively studied (Dember \& Richman, 1989).

Another method of accessing spontaneous alternation is with a Y-maze (with three equally spaced arms) or a plusmaze (with four equally spaced arms); the rat (or mouse) is allowed to freely explore the maze, and alternation of arm choices is assessed. This has been called continuous spontaneous alternation (Drew, Kostas, McFarland, \& de Rossett, 1980; Drew, Miller, \& Baugh, 1973) to distinguish it from T-maze spontaneous alternation (which is typically tested with discrete pairs of trials). Alternation in a Y-maze under such conditions was first reported by Dennis and Sollenberger (1934) and later became popular for use in pharmacological studies of memory in rodents (Drew et al., 1973; Lennartz \& Gold, 1995; Ragozzino \& Gold, 1994; Sarter, Bodewitz, \& Stephens, 1988; D. L. Walker \& Gold, 1991). The usual method of determining alternation scores in these studies is to evaluate overlapping runs of three choices and then calculate the number of alternations (entries into three different arms) as a percentage of the total possible number of alternations. For example, if the three arms are designated $\mathrm{A}, \mathrm{B}$, and $\mathrm{C}$, the choices $\mathrm{CA} B$ A $C$ A $B C$ B $A C B$ would contain seven alternation sequences (the letters in italics represent the ends of these sequences). Thus, starting at the beginning of the run of choices, $\mathrm{CAB}$ is considered an alternation sequence, but ABA is not. In this case, the total number of possible alternations is 10 (the number of choices minus two), and thus the alternation score is $70 \%$.

More recently, a plus-maze version has been used (McNay, Fries, \& Gold, 2000; Ragozzino, Pal, Unick, Stefani, \& Gold, 1998; Ragozzino, Unick, \& Gold, 1996). From a theoretical viewpoint, this would appear to be a more difficult task than alternation in a Y-maze. A rat emerging from an arm in the Y-maze must remember which of two arms was last visited in order to demonstrate alternation, but a rat emerging from an arm in the plus-maze must remember which one of three arms was least recently visited. McNay et al. offered neurobiological data that supported this idea that the latter task is more cognitively demanding. They showed that rats exhibit a larger decrease in extracellular glucose in the hippocampus, a structure that is important for spatial memory (Morris, Garrud, Rawlins, \& O'Keefe, 1982; Olton, Walker, \& Gage, 1978), in the plus-maze, as compared with the Y-maze, suggesting that the hippocampus is more active (and thus requires more glucose) during the plus-maze alternation.

Performance in other spatial tasks, such as the radial arm maze (Olton \& Samuelson, 1976) and the Morris water maze (Morris, 1981), are sensitive to manipulations of extramaze cues - cues located in the environment outside the apparatus (Baldi, Lorenzini, \& Bucherelli, 2003; Diez-Chamizo, Sterio, \& Mackintosh, 1985; Sutherland \& Dyck, 1984; Suzuki, Augerinos, \& Black, 1980). For example, Suzuki et al. showed that learning in the radial arm maze was impaired when such cues were absent, although performance was greater than chance in the stimuluspoor condition. Furthermore, rotation of the cues was less detrimental to performance than was transposition of the cues. The authors suggest that these data demonstrate that

R. C. Lennartz, rlennart@ius.edu 
it is the configuration of cues that the rat uses to navigate the maze.

The idea that animals use an array of cues in finding a goal in spatial tasks supports Tolman's (1948) idea that rats construct a cognitive map of their environment in such tasks. This idea was further developed by O'Keefe and Nadel (1978), who referred to the use of various stimuli to locate a goal as "locale learning" and claimed that this is different from associative learning (such as learning about the relationship between a single stimulus and a goal). Although the use of distal cues by rats in spatial tasks is well established, there have been alternative explanations to the cognitive map account. For example, it has been suggested that room cues provide direction information, rather than location information (Hamilton, Akers, Weisend, \& Sutherland, 2007). Other researchers have argued that the use of distal cues to find a goal does follow the same rules as associative learning, rather than being part of a specialized locale system (for reviews, see Chamizo, 2003; Mackintosh, 2002).

There are no published data on the use of extramaze cues by rats in a continuous alternation task. It appears that numerous cues can be used for spontaneous alternation in a T-maze; the relative salience of the various stimuli may determine what is important (E. L. Walker, Dember, Earl, \& Karoly, 1955), a conclusion also reached by Restle (1957) using a rewarded alternation task. However, extramaze cues do not appear to have been as systematically or carefully studied as other cues in T-maze spontaneous alternation, and as has been noted, no data have been published on the role extramaze cues play in continuous spontaneous alternation.

One common feature of the continuous spontaneous alternation, radial arm maze, and Morris water maze tasks is that the rodent explores and learns about an environment. However, in the latter two tasks, learning is assessed by the rat's ability to find explicit goals (food or escape from water), with the extramaze cues providing information about the location. Spontaneous alternation is a form of exploratory behavior (Dember \& Richman, 1989; Hughes, 1998), and there are no explicit rewards; learning is revealed by the rat's demonstrating a search strategy in which recently visited locations are not immediately revisited. What role do extramaze cues play in spatial tasks in which the rat is not remembering the location of reinforcers in relation to these cues? That rats do learn about the maze environment without reward has been shown in work on latent learning (Blodgett, 1929; Tolman \& Honzik, 1930) and with other types of preexposure effects in spatial memory (for a review, see Prados \& Redhead, 2002). In all of these tasks, learning during the preexposure phase is assessed in a second phase in which the goal (food or a platform) is introduced. In a sense, spontaneous alternation allows a more direct assessment of spatial learning during nonrewarded exploration and can serve as an approach by which to test the role of extramaze cues in a situation in which there are no specific reinforcers for which the rat is searching.

One purpose of the present experiment was to systematically address the issue of the effects of extramaze cues on continuous spontaneous alternation in a plus-maze. A technique similar to that in Suzuki et al. (1980) was adopted. A curtain was placed around the maze, allowing rats to be tested without cues or with cues (by placing stimuli on the curtain). In addition, a third group was tested in the dark, essentially removing all visual cues. If extramaze cues are beneficial to performance in this task, as they are with the other spatial tasks, alternation should be best in the stimulus-rich condition. Data such as these can provide a better understanding of the cues that are used in spatial navigation; in addition, such a behavioral analysis may be useful in the interpretation of the neurobiological data obtained when animals are tested on this task.

A second purpose of this study was to investigate the effects of daily testing on alternation performance. In other spatial tasks, reinforcement maintains the behavior; there is no reinforcement in spontaneous alternation. Although habituation of activity may be expected when a rat is placed repeatedly into the same environment (Howarth, 1962), there are few data available on what happens to alternation performance in a continuous alternation task. In the majority of such studies, the researchers have tested the rodents only on single sessions; there have been few studies spanning multiple consecutive days. With 2 days of daily sessions in mice, alternation did significantly decrease on the second day (Kokkinidis \& Anisman, 1976a) but was still above chance. The rats in the present study were tested in five daily sessions in order to assess changes in alternation and also to test for interactions between day of testing and cue density.

\section{METHOD}

\section{Subjects}

The subjects were male Sprague Dawley rats (Harlan Sprague Dawley, Indianapolis, IN) approximately 60 days old at the time of arrival. They were housed on a 12:12-h light:dark cycle (lights on at 8:00 a.m.), with food and water freely available. The rats were handled for 2 min per day for 4 days the week of arrival, and testing started 1-2 weeks after arrival.

\section{Apparatus}

The translucent Plexiglas maze (Med Associates, St. Albans, VT) consisted of an octagon-shaped central hub $(33 \mathrm{~cm}$ in height and $30.5 \mathrm{~cm}$ between opposing sides) with four arms radiating from it at $90^{\circ}$ angles to one another. Each arm was $61 \mathrm{~cm}$ in length, $9 \mathrm{~cm}$ in width, and $16.8 \mathrm{~cm}$ in height (inside dimensions). A Plexiglas lid covered each arm. The metal panels in the four sides of the hub without arms were replaced with translucent Plexiglas to facilitate viewing of the extramaze cues by the rats.

Each arm contained three infrared (IR) source/detector pairs (Med Associates). Two pairs were located near the entrance of each arm (5.1 and $7.3 \mathrm{~cm}$ from the hub), and one pair was located at the midpoint; all were $3.8 \mathrm{~cm}$ from the maze floor. Data on arm entries were collected via software (MED-PC Version IV; Med Associates) run on a Dell computer.

The maze was located on a round table, $152.4 \mathrm{~cm}$ in diameter. The table was surrounded by a black fabric curtain that was attached to a hoop mounted to the walls of the room. The bottom of the curtain was approximately $20 \mathrm{~cm}$ below the tabletop. Safety pins inserted into the curtain allowed the attachment of stimuli. The top of the hoop was covered by another piece of dark fabric to form a false ceiling (approximately $1.3 \mathrm{~m}$ above the table) to block out cues on the ceiling. A hole was cut into the middle of the false ceiling, a light (23-W spiral fluorescent bulb with output equivalent to a 100-W incandescent bulb) was mounted above this, and a semiopaque piece of plastic was inserted into the hole. All other room lights were off during testing. A PC camera (QuickCam Pro 4000, Logitech) was mounted near the light and focused on the central hub and arm entrances. 
The computer for collection of data (from the infrared detectors and video camera) was located in the room, just outside the curtain.

\section{Procedure}

Each rat was carried from the animal room to the maze room in its home cage, with a towel covering the cage to minimize exposure to visual cues between leaving the animal room and being placed into the maze. The cage was placed on the floor near the maze, and the rat was removed, placed into the arm near the opening in the curtain, and released. The lid was placed on the arm, the curtain was fastened shut, and the experimenter left the room and closed the door. After $12 \mathrm{~min}$, the rat was retrieved and returned to the animal room, and the maze floor was cleaned with a $70 \%$ alcohol solution. Each rat was tested on 5 consecutive days. All the animals were tested between 8:15 a.m. and 12:30 p.m.

An arm entry was counted if the rat broke the two beams near the hub and subsequently broke the beam at the midpoint of the arm. Preliminary testing revealed that the system accurately detected arm entries, with one exception. Occasionally, a double entry (i.e., two consecutive entries into the same arm) was falsely recorded if the rat, after breaking the beam near the midpoint, reared in the arm near the hub and then turned around and broke the midpoint beam again. Because of this, all double entries - whether true (as when a rat completely leaves an arm and then reenters it before choosing another arm) or false - recorded by the IR system were not used in the calculation of spontaneous alternation. The exclusion of immediate reentries into arms in alternation calculations is not unprecedented in Y-maze studies (Anisman, 1975; Kokkinidis \& Anisman, 1976b). Furthermore, although it is not clear from the method descriptions in the plus-maze studies as to whether immediate reentries were counted, the calculation of chance performance as $44.4 \%$ (McNay \& Gold, 2001) is consistent with this approach. (See the Appendix for an explanation of the calculation of chance performance for this task.)

For the present data, the frequency of double entries (presumably including both real and false reentries) as a function of total number of entries was calculated for each session for each rat; the overall mean was $7.5 \%(S D=4.7 \%)$.

For additional analyses from the video data, an arm entry was counted if the rat entered an arm with all four feet, which is a typical criterion used in this research (Anisman, 1975; D. L. Walker \& Gold, 1994).

Alternation was calculated using the method of Ragozzino et al. (1996). An alternation was counted if in five consecutive arm choices, the rat chose four different arms. Overlapping runs of five choices were used; the total possible number of alternations was the number of choices minus 4 . Percentage of alternation was calculated as the number of actual alternations divided by the number of possible alternations and then multiplied by 100 . For example, if the choices were D B C A $B$ C A $D A$ C $B D$, there are five sequences that count as alternations (the italicized letters indicate the end of a run containing an alternation). Starting at the beginning of the run of choices, DBCAB is an alternation because all four arms were visited, but $\mathrm{BCABC}$ is not considered an alternation run. The total possible number of alternations in this case is eight ( 12 choices minus 4 ), and so the alternation score would be $5 / 8$ or $62.5 \%$.

It should be noted that in spontaneous alternation in a Y-maze, alternation is most commonly assessed by evaluating overlapping runs of three arms. Thus, for the plus-maze, analyzing the data on the basis of sequences of four choices, rather than five, would be a closer parallel to the Y-maze technique. The four different arms in five choices procedure was used to assess alternation performance for the data presented here because this is the analysis used in previously published studies of continuous alternation in a plus-maze. However, additional analyses based on runs of four choices yielded the same general results.

The rats were randomly assigned to one of three groups. For the dark group $(n=11)$, the overhead light above the false ceiling was turned off. When the rats were retrieved from this group after testing, a red light was used. The other two groups were designated as stimulus poor and stimulus rich, using the terminology of Suzuki et al. (1980).
For the stimulus-poor group $(n=12)$, the overhead light was on, but no cues were attached to the curtain. For the stimulus-rich group $(n=$ 11), the overhead light was on, and 10 stimuli were attached to the curtain. Five of the stimuli were shapes cut from white cardboard. The shapes were a star $(55.9 \mathrm{~cm}$ in height and $61 \mathrm{~cm}$ in width), a rectangle $(71.1 \mathrm{~cm}$ in height and $55.9 \mathrm{~cm}$ in width), a circle $(53.3 \mathrm{~cm}$ in diameter), a triangle (55.2 $\mathrm{cm}$ in height and $71.1 \mathrm{~cm}$ across the base), and an irregular, roughly plus-shaped figure with rounded edges $(64.8 \mathrm{~cm}$ in height and $54 \mathrm{~cm}$ in width). The other 5 stimuli were a yellow oval hoop $(52.1 \mathrm{~cm}$ in height and $47 \mathrm{~cm}$ in width; the hoop itself was constructed from a foam tube $19.1 \mathrm{~cm}$ in circumference), a small plastic kite $(56.5 \mathrm{~cm}$ in height and $54.6 \mathrm{~cm}$ in width), a large plastic ball (106.7 $\mathrm{cm}$ in circumference), a black-and-white poster $(91.4 \mathrm{~cm}$ in height and $30.5 \mathrm{~cm}$ in width), and a large decorative butterfly $(49.5 \mathrm{~cm}$ in height and $38.1 \mathrm{~cm}$ in width). The positions of the stimuli are indicated in Figure 1. The heights of these stimuli from the table varied, but all were located between the table and the false ceiling.

\section{RESULTS}

\section{Spontaneous Alternation and Activity on the First Day}

Because testing on continuous spontaneous alternation is typically assessed in a single session, the initial analyses were restricted to the first day. Figure 2 shows the alternation scores for the three groups of animals; the scores for the stimulus-rich group are higher than those for the dark and stimulus-poor groups. A one-way ANOVA revealed that there was a significant difference among the groups $[F(2,31)=5.281, p=.011]$, and a post hoc Tukey HSD analysis was conducted. The stimulus-rich group alternated significantly more than did the stimulus-poor group ( $p=$ .010). The difference between the stimulus-rich group and the dark group approached significance $(p=.069)$.

Chance performance, indicated in Figure 2, is $44.4 \%$ (see the Appendix). One-sample $t$ tests were used to compare first-day alternation scores with this value; the scores for all three groups were significantly above chance (all $p$ values $\leq$ $.001)$. Thus, even the rats tested in the dark, with no visual cues, alternated at a level above that expected by chance.

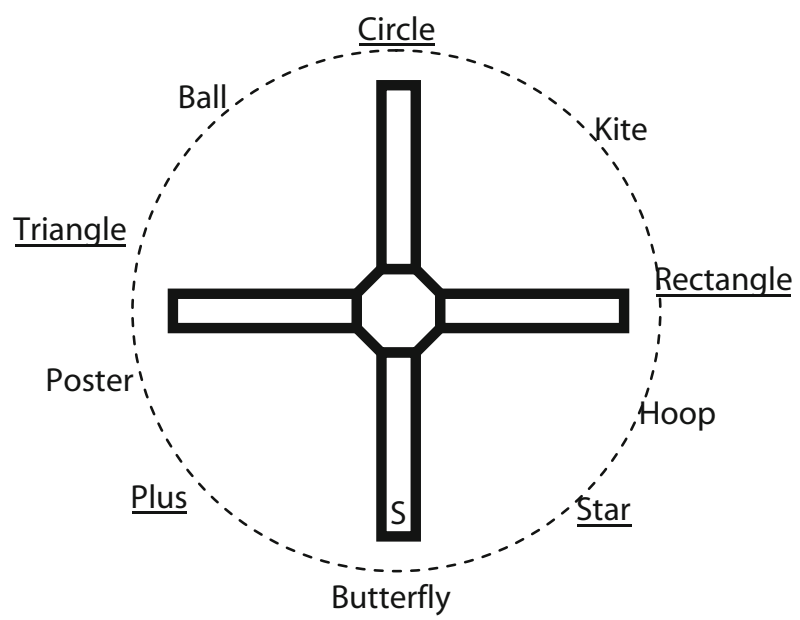

Figure 1. The positions of the extramaze stimuli relative to the maze arms. The underlining indicates stimuli that were constructed from white cardboard. The " $S$ " is the point at which the rat was initially placed. The stimuli are described in the text. 


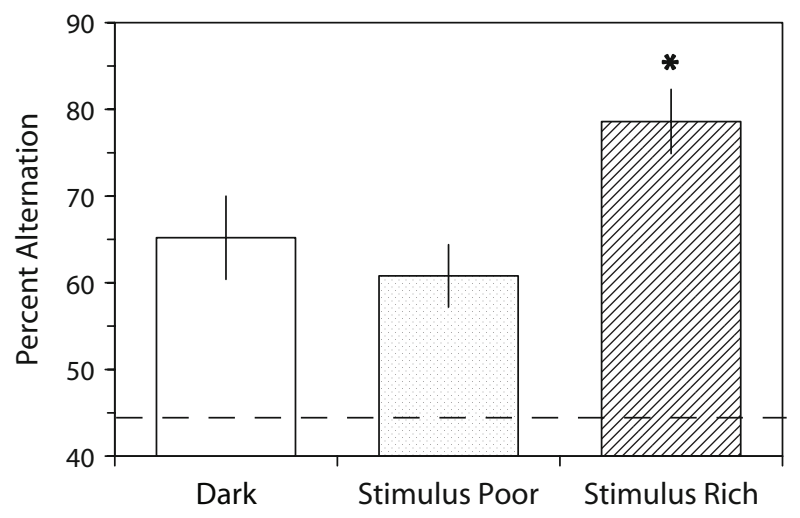

Figure 2. The mean spontaneous alternation scores for the dark, stimulus-poor, and stimulus-rich groups on the first day of testing. The error bars represent the $S E M$ s. The dotted line is at the level of chance alternation. *Statistically significant difference $(p=.010)$, as compared with the stimulus-poor group.

As a measure of activity in the maze, an analysis was also performed on the number of arm choices. The mean values were $30.7(S D=3.5)$ for the dark group, $33.4(S D=$ $6.9)$ for the stimulus-poor group, and $27.7(S D=6.3)$ for the stimulus-rich group. A one-way ANOVA revealed that the difference in number of arm choices among the groups approached significance $[F(2,31)=2.787, p=.077]$. To determine the source of this marginal significance, a post hoc Tukey HSD analysis was conducted. The difference between the stimulus-rich and the stimulus-poor groups approached significance $(p=.062)$.

\section{Spontaneous Alternation and Activity Across Days}

Figure 3 shows the alternation scores for the three groups over the 5 days. The alternation scores for the stimulus-rich group appeared to fall over the 5 days, whereas those for the other two groups increased. A two-way mixed ANOVA, with group (extramaze cue condition) as the betweensubjects variable and day as the within-subjects variable, was conducted. There was no main effect of condition $[F(2,31)=0.411, p=.667]$ or of day $[F(4,124)=1.980$, $p=.102]$. However, there was a significant group $\times$ day interaction $[F(8,124)=3.307, p=.002]$.

A trend analysis repeated measures ANOVA was used to test for patterns across days in each group. There was a significant linear trend for both the stimulus-poor group $[F(1,11)=6.505, p=.027]$ and the stimulus-rich group $[F(1,10)=6.219, p=.032]$. As can be seen in Figure 3, alternation scores for the stimulus-poor group increased over days, and those for the stimulus-rich group tended to decrease over days. There were no significant trends over days for the dark group.

Figure 4 shows the number of arm choices for the three groups over the 5 days (based on the choices detected by the IR system). A two-way mixed ANOVA, with group (extramaze cue condition) as the between-subjects variable and day as the within-subjects variable, was conducted. Due to the violation of sphericity, the Huynh-Feldt procedure was used to adjust the degrees of freedom for the within-subjects and interaction analyses. There was no main effect of condition $[F(2,31)=0.079, p=.924]$, but there was a main effect of day $[F(3.76,116.69)=2.538$, $p=.047]$. There was a significant group $\times$ day interaction $[F(7.53,116.69)=2.574, p=.014]$.

A trend analysis repeated measures ANOVA was used to test for patterns across days in each group. There was a significant linear trend for the stimulus-rich group $[F(1,10)=$ $20.172, p=.001]$, and there was also a significant quadratic component $[F(1,10)=6.709, p=.027]$. As can be seen in Figure 4, the number of choices for the stimulus-rich group increased, then fell and leveled off. There were no significant trends over days for the stimulus-poor or the dark group.

\section{Spontaneous Alternation Determination With a Less Stringent Criterion for Arm Choice}

The criterion for arm entry with the data collected via the IR system in this study was that the rat must enter an arm at least halfway. This was stricter than the criterion that has often been used with continuous alternation, which is entrance of all four feet into an arm (Anisman, 1975; D. L. Walker \& Gold, 1994). The video data for the stimulus-rich and stimulus-poor groups were analyzed using this criterion. One rat from the stimulus-poor group was dropped from this analysis because there were no video data on the fourth day, due to experimenter error. Overall, the same main conclusions can be made regardless of which criterion for an arm choice is used.

\section{DISCUSSION}

On the first day of testing, the rats in the stimulus-rich condition alternated more than did the rats in the stimuluspoor condition (and marginally significantly more than the rats in the dark condition), supporting the idea that extramaze cues enhance performance on this spatial task as they do on other spatial tasks. However, on the 4 subsequent days, this group did not show superior alternation performance. There was a tendency for the rats in the stimulus-poor group to increase alternation over days and an opposite trend for the stimulus-poor rats.

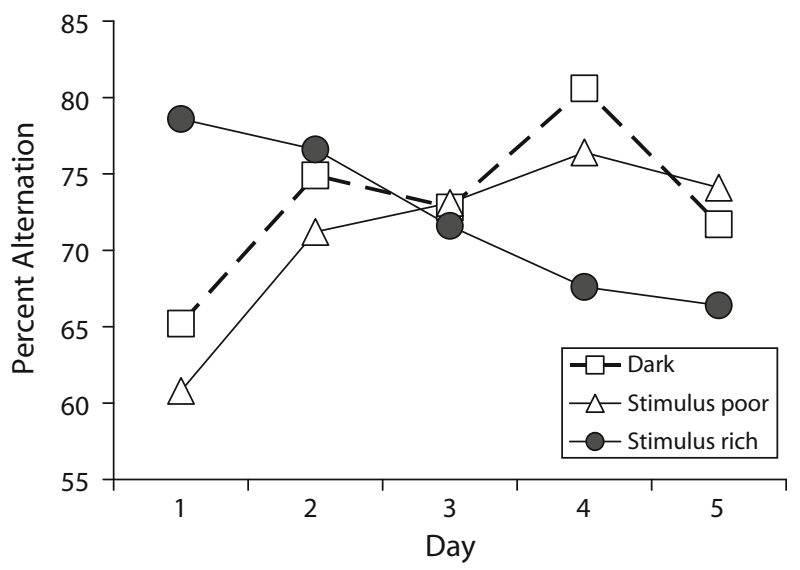

Figure 3. The mean spontaneous alternation scores across days for each of the three groups. 


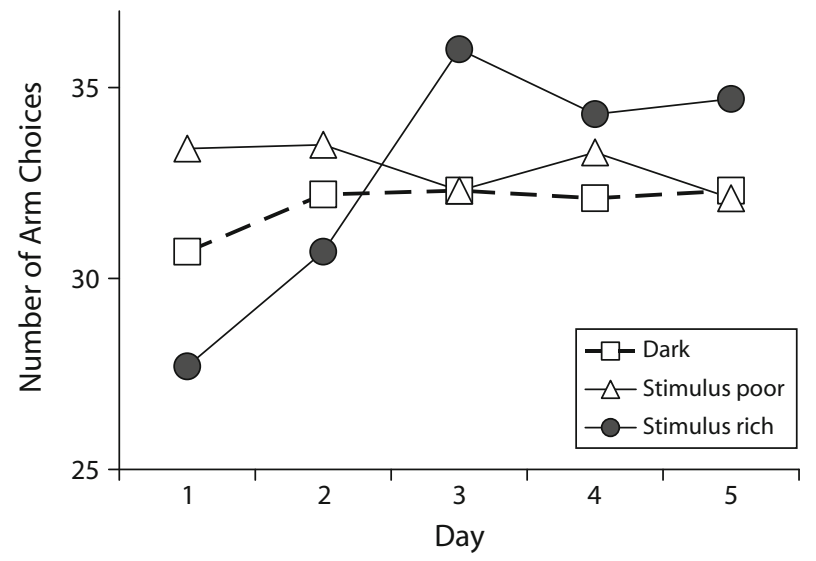

Figure 4. The mean number of arm entries (not including immediate reentries into an arm just visited) across days for each of the three groups.

In most spatial tasks, when extramaze cues are present, the rat appears to learn about the location of rewards in relation to these cues (Baldi et al., 2003; Diez-Chamizo et al., 1985; Sutherland \& Dyck, 1984; Suzuki et al., 1980). The present study indicates that, at least on the first day of testing, such cues assisted optimal exploration (defined here as not revisiting arms that were recently visited) of the maze in the absence of reinforcers. The lower activity (as measured by number of arm choices) of the stimulus-rich rats, as compared with the stimulus-poor rats, on this first day could possibly be due to the rats' learning about the extramaze stimuli and may be indicative of perceptual learning (Gibson, 1969).

Although the presence of extramaze cues enhanced performance in the first session, it is clear from the present data that extramaze cues are not the only cues used by rats in this task. All groups, including the rats tested in total darkness, alternated at levels above chance on all days. Suzuki et al. (1980) also found in the radial arm maze that although rats performed better in the presence of extramaze cues, they still performed above chance in their absence.

Auditory stimuli are, potentially, another type of extramaze cue that could be used in guiding spatial behavior. In the present experiment, there was a computer near the curtain and an air vent in the ceiling of one corner of the room. However, Douglas $(1964,1966 a)$ found no evidence that auditory cues are used in T-maze alternation. In the Morris water maze, Sutherland and Dyck (1984) found that a buzzer and metronome could support spatial learning in both enucleated and sighted rats; however, Rossier, Haeberli, and Schenk (2000) found that three auditory cues are not sufficient for good spatial learning in rats trained in the dark.

Intramaze visual cues - stimuli within the apparatusare another possible source of guidance for rats. Although it has been demonstrated in T-maze alternation that prominent intramaze visual cues can support alternation (Eisenberger, Myers, Sanders, \& Shanab, 1970; Pate \& DeLoache, 1970; E. L. Walker et al., 1955), such stimuli can be ruled out for the dark group in the present experiment. Although subtle intramaze visual cues could have been used by the rats in the other two groups, there was no significant difference in alternation between the dark group and the stimulus-poor group, suggesting that the rats in the latter group did not benefit from cues unavailable to the dark group. It should also be noted that in the radial arm maze, when explicit visual intramaze cues are provided, rats do not appear to use them (Babb \& Crystal, 2003).

The evidence for use of intramaze olfactory cues in T-maze alternation is equivocal, with data both supporting (Douglas, 1966a; Klein \& Brown, 1969; Rosen \& Stein, 1969) and not supporting (Douglas, Isaacson, \& Moss, 1969; Richman, Gulkin, \& Knoblock, 1972) their use. Theoretically, such cues would be more straightforward in the T-maze version than in the continuous version. In the former, the rat could simply avoid the arm with the odor trail left during the first visit. In continuous alternation in a plus-maze (or Y-maze), there would potentially be multiple odor trails, requiring the rat to distinguish between the most recent and earlier odors. Olton and Collison (1979) showed that rats perform poorly in the radial arm maze when olfactory cues are the only ones that they can use.

Navigation by use of external cues, or piloting, is not the only method available to animals; they can also use dead reckoning (path integration), in which cues generated internally by the animal's movements help guide the animal (Gallistel, 1990). Interestingly, the use of dead reckoning has been implicated in a form of spontaneous exploratory behavior; rats will return by a direct path to a home site after exploration of a circular surface even in the absence of light (Whishaw, Hines, \& Wallace, 2001). One suggested cue for dead reckoning is vestibular information (Mittelstaedt \& Mittelstaedt, 1980). More recent data obtained from rats with labyrinthectomies support the idea that this information is important for navigation by dead reckoning in a task in which rats forage for food pellets (Wallace, Hines, Pellis, \& Whishaw, 2002). Although this process has been studied mainly in tasks in which the animal leaves a home site and returns to it, there is evidence that at least vestibular information may be a relevant cue in spontaneous alternation in a T-maze (Douglas, 1966a, 1966b; Franken \& Baker, 1969), as well as in the radial arm maze (Dudchenko, Goodridge, Seiterle, \& Taube, 1997; Ossenkopp \& Hargreaves, 1993). There have been no studies on the role of such information in continuous spontaneous alternation.

In addition to investigating the use of extramaze cues in a continuous spontaneous alternation task, another purpose of the present study was to investigate changes in alternation behavior over days; this is the first known study in which continuous spontaneous alternation testing was performed repeatedly on a daily basis. In discrete trial alternation in a T-maze, Douglas (1964) found no change in alternation with repeated testing. In the Y-maze, a decrease was found when mice were tested on 2 consecutive days (Kokkinidis \& Anisman, 1976a). In the present investigation involving daily sessions in a plus-maze, the nature of the changes depended on the cue condition. There were significant trends of an increase in alternation for the stimulus-poor group and a decrease in alternation for the stimulus-rich group. Although the trend for the dark group was not significant, it showed a pattern similar to that of the stimulus-poor group (Figure 3 ). One possibility is that with repeated exposure to the maze in the absence 
of salient extramaze cues, the rats became more efficient in discriminating and using other types of cues, perhaps again demonstrating perceptual learning (Gibson, 1969).

The decrease in alternation for the stimulus-rich condition could indicate either that the extramaze cues were less attended to over days or that the cues were still used but that alternation decreased for other reasons. One possible way to distinguish between these two interpretations would be to remove the cues for the stimulus-rich group on one of the later sessions; if there is a sudden drop in alternation scores, it could indicate that the extramaze cues were still at least partially supporting alternation in these rats.

Neurobiological studies of plus-maze alternation have focused on hippocampal functioning (McNay et al., 2000; Ragozzino et al., 1998; Ragozzino et al., 1996; Stefani \& Gold, 2001). One role of the hippocampus in spatial behavior may be in the formation of cognitive maps of the environment (Morris et al., 1982; O'Keefe \& Nadel, 1978); such maps are thought to incorporate the available extramaze cues. However, as has been shown here, when such cues are present they may have a limited role in alternation in a plus-maze, and rats alternate well above chance even in the absence of any visual information. Of course, the hippocampus may play other roles in spatial behavior in addition to cognitive map formation. For example, there is also evidence that the hippocampus is required for dead reckoning, both in situations involving the retrieval of food pellets and in spontaneous exploration of a circular surface (Whishaw et al., 2001).

In conclusion, the present study supports the idea that, as with other spatial tasks, performance in a continuous nonrewarded spontaneous alternation task is better in an environment rich in extramaze cues but this superiority is observed only on the initial testing day. In addition, and similar to results reported for the radial arm maze (Suzuki et al., 1980), performance in the absence of extramaze cues is still above chance. Thus, other cues, possibly internally generated, must also be guiding the alternation performance in the plus-maze.

\section{AUTHOR NOTE}

The author thanks Gary Bamberger of Med Associates, Inc., for writing the data collection program for the infrared system. Correspondence concerning this article should be addressed to R. C. Lennartz, Department of Psychology, Indiana University Southeast, 4201 Grant Line Road, New Albany, IN 47150 (e-mail: rlennart@ius.edu).

\section{REFERENCES}

Anisman, H. (1975). Dissociation of disinhibitory effects of scopolamine: Strain and task factors. Pharmacology, Biochemistry, \& Behavior, 3, 613-618.

BABB, S. J., \& CRYSTAL, J. D. (2003). Spatial navigation on the radial maze with trial-unique intramaze cues and restricted extramaze cues. Behavioural Processes, 64, 103-111.

Baldi, E., Lorenzini, C. A., \& Bucherelli, C. (2003). Task solving by procedural strategies in the Morris water maze. Physiology \& Behavior, 78, 785-793.

BLodgetT, H. C. (1929). The effect of the introduction of reward upon the maze performance of rats. University of California Publications in Psychology, 4, 113-134.

Chamizo, V. D. (2003). Acquisition of knowledge about spatial location: Assessing the generality of the mechanism of learning. Quarterly Journal of Experimental Psychology, 56B, 102-113.
Dember, W. N., \& Richman, C. L. (Eds.) (1989). Spontaneous alternation behavior. New York: Springer.

DenNIs, W. (1935). A comparison of the rat's first and second explorations of a maze unit. American Journal of Psychology, 47, 488-490.

Dennis, W., \& Sollenberger, R. T. (1934). Negative adaptation in the maze exploration of rats. Journal of Comparative Psychology, 18, 197-206.

Diez-Chamizo, V., Sterio, D., \& Mackintosh, N. J. (1985). Blocking and overshadowing between intra-maze and extra-maze cues: A test of the independence of locale and guidance learning. Quarterly Journal of Experimental Psychology, 37B, 235-253.

Douglas, R. J. (1964). An analysis of spontaneous alternation cues. Dissertation Abstracts International, 25(12), 7388. (UMI No. AAT 6505297)

Douglas, R. J. (1966a). Cues for spontaneous alternation. Journal of Comparative \& Physiological Psychology, 62, 171-183.

Douglas, R. J. (1966b). Spontaneous alternation and middle ear disease. Psychonomic Science, 4, 243-244.

Douglas, R. J., IsaAcson, R. L., \& Moss, R. L. (1969). Olfactory lesions, emotionality and activity. Physiology \& Behavior, 4, 379-381.

Drew, W. G., Kostas, J., McFarland, D. J., \& de Rossett, S. E. (1980). Continuous, spontaneous alternation in the rat: Influence of Y-maze arm times. Perceptual \& Motor Skills, 51, 359-369.

Drew, W. G., Miller, L. L., \& BaUgh, E. L. (1973). Effects of $\Delta$ 9-THC, LSD-25 and scopolamine on continuous, spontaneous alternation in the Y-maze. Psychopharmacologia, 32, 171-182.

Dudchenko, P. A., Goodridge, J. P., Seiterle, D. A., \& Taube, J. S. (1997). Effects of repeated disorientation on the acquisition of spatial tasks in rats: Dissociation between the appetitive radial arm maze and aversive water maze. Journal of Experimental Psychology: Animal Behavior Processes, 23, 194-210.

Eisenberger, R., Myers, A. K., Sanders, R., \& Shanab, M. (1970). Stimulus control of spontaneous alternation in the rat. Journal of Comparative \& Physiological Psychology, 70, 136-140.

FranKen, R. E., \& BAKER, J. G. (1969). The effects of drive level on cues utilized in spontaneous alternation. Psychonomic Science, 16, 239-240.

Gallistel, C. R. (1990). The organization of learning. Cambridge, MA: MIT Press.

Gibson, E. J. (1969). Principles of perceptual learning and development. New York: Appleton-Century-Crofts.

Hamilton, D. A., Akers, K. G., Weisend, M. P., \& Sutherland, R. J. (2007). How do room and apparatus cues control navigation in the Morris water task? Evidence for distinct contributions to a movement vector. Journal of Experimental Psychology: Animal Behavior Processes, 33, 100-114.

HowARTH, E. (1962). Activity decrements and recovery during repeated day to day exposure to the same environment. Journal of Comparative \& Physiological Psychology, 55, 1102-1104.

HugHES, R. N. (1998). Spontaneous alternation behavior in animals: Mechanisms, motives, and applications. In R. R. Hoffman, M. F. Sherrick, \& J. S. Warm (Eds.), Viewing psychology as a whole: The integrative science of William N. Dember (pp. 269-286). Washington, DC: American Psychological Association.

KLEIN, D., \& BROWN, T. S. (1969). Exploratory behavior and spontaneous alternation in blind and anosmic rats. Journal of Comparative \& Physiological Psychology, 68, 107-110.

KoKKINIDIS, L., \& ANISMAN, H. (1976a). Dissociation of the effects of scopolamine and d-amphetamine on a spontaneous alternation task. Pharmacology, Biochemistry, \& Behavior, 5, 293-297.

KoKkinidis, L., \& ANISMAN, H. (1976b). Interaction between cholinergic and catecholaminergic agents in a spontaneous alternation task. Psychopharmacology, 48, 261-270.

LenNaRTZ, R. C., \& Gold, P. E. (1995). Glucose does not reverse impairments on spontaneous alternation induced by the noncompetitive NMDA antagonist MK-801. Neurobiology of Learning \& Memory, 63, $107-110$.

Mackintosh, N. J. (2002). Do not ask whether they have a cognitive map, but how they find their way about. Psicológica, 23, 165-185.

McNay, E. C., Fries, T. M., \& GolD, P. E. (2000). Decreases in rat extracellular hippocampal glucose concentration associated with cognitive demand during a spatial task. Proceedings of the National Academy of Sciences, 97, 2881-2885. 
McNay, E. C., \& Gold, P. E. (2001). Age-related differences in hippocampal extracellular fluid glucose concentration during behavioral testing and following systemic glucose administration. Journals of Gerontology, 56A, B66-B71.

Mittelstaedt, M.-L., \& Mittelstaedt, H. (1980). Homing by path integration in a mammal. Naturwissenschaften, 67, 566-567.

Morris, R. G. M. (1981). Spatial localization does not require the presence of local cues. Learning \& Motivation, 12, 239-260.

Morris, R. G. M., Garrud, P., Rawlins, J. N., \& O'Keefe, J. (1982) Place navigation impaired in rats with hippocampal lesions. Nature, 297, 681-683.

O'KeEFE, J., \& NADEL, L. (1978). The hippocampus as a cognitive map. Oxford: Oxford University Press, Clarendon Press.

Olton, D. S., \& Collison, C. (1979). Intramaze cues and "odor trails" fail to direct choice behavior on an elevated maze. Animal Learning \& Behavior, 7, 221-223.

Olton, D. S., \& Samuelson, R. J. (1976). Remembrance of places passed: Spatial memory in rats. Journal of Experimental Psychology: Animal Behavior Processes, 2, 97-116.

Olton, D. S., Walker, J. A., \& Gage, F. H. (1978). Hippocampal connections and spatial discrimination. Brain Research, 139, 295-308.

Ossenkopp, K.-P., \& Hargreaves, E. L. (1993). Spatial learning in an enclosed eight-arm radial maze in rats with sodium arsanilate-induced labyrinthectomies. Behavioral \& Neural Biology, 59, 253-257.

Pate, J. L., \& DeLoache, J. (1970). Brightness and direction as cues for spontaneous alternation behavior. Psychonomic Science, 18, 27-28.

Prados, J., \& Redhead, E. S. (2002). Preexposure effects in spatial learning: From gestaltic to associative and attentional cognitive maps. Psicológica, 23, 59-78.

Ragozzino, M. E., \& Gold, P. E. (1994). Task-dependent effects of intra-amygdala morphine injections: Attenuation by intra-amygdala glucose injections. Journal of Neuroscience, 14, 7478-7485.

Ragozzino, M. E., Pal, S. N., Unick, K., Stefani, M. R., \& Gold, P. E. (1998). Modulation of hippocampal acetylcholine release and spontaneous alternation scores by intrahippocampal glucose injections. Journal of Neuroscience, 18, 1595-1601.

Ragozzino, M. E., Unick, K. E., \& Gold, P. E. (1996). Hippocampal acetylcholine release during memory testing in rats: Augmentation by glucose. Proceedings of the National Academy of Sciences, 93, 4693-4698.

Restle, F. (1957). Discrimination of cues in mazes: A resolution of the "place-vs.-response" question. Psychological Review, 64, 217-228.

Richman, C. L., Gulkin, R., \& Knoblock, K. (1972). Effects of bulbectomization, strain, and gentling on emotionality and exploratory behavior in rats. Physiology \& Behavior, 8, 447-452.
Rosen, J. J., \& STEIN, D. G. (1969). Spontaneous alternation behavior in the rat. Journal of Comparative \& Physiological Psychology, 68, 420-426

Rossier, J., Haeberli, C., \& Schenk, F. (2000). Auditory cues support place navigation in rats when associated with a visual cue. Behavioural Brain Research, 117, 209-214.

Sarter, M., Bodewitz, G., \& Stephens, D. N. (1988). Attenuation of scopolamine-induced impairment of spontaneous alternation behaviour by antagonist but not inverse agonist and agonist $\beta$-carbolines. Psychopharmacology, 94, 491-495.

Stefani, M. R., \& Gold, P. E. (2001). Intrahippocampal infusions of K-ATP channel modulators influence spontaneous alternation performance: Relationships to acetylcholine release in the hippocampus. Journal of Neuroscience, 21, 609-614.

Sutherland, R. J., \& DYcK, R. H. (1984). Place navigation by rats in a swimming pool. Canadian Journal of Psychology, 38, 322-347.

Suzuki, S., Augerinos, G., \& Black, A. H. (1980). Stimulus control of spatial behavior on the eight-arm maze in rats. Learning \& Motivation, 11, 1-18.

Tolman, E. C. (1925). Purpose and cognition: The determiners of animal learning. Psychological Review, 32, 285-297.

Tolman, E. C. (1948). Cognitive maps in rats and men. Psychological Review, 55, 189-208.

Tolman, E. C., \& HonzIK, C. H. (1930). Degrees of hunger, reward and non-reward, and maze learning in rats. University of California Publications in Psychology, 4, 241-256.

Walker, D. L., \& Gold, P. E. (1991). Effects of the novel NMDA antagonist, NPC 12626, on long-term potentiation, learning and memory. Brain Research, 549, 213-221.

WALKER, D. L., \& GoLD, P. E. (1994). Intrahippocampal administration of both the D- and the L-isomers of AP5 disrupt spontaneous alternation behavior and evoked potentials. Behavioral \& Neural Biology, 62, 151-162.

Walker, E. L., Dember, W. N., Earl, R. W., \& Karoly, A. J. (1955). Choice alternation: I. Stimulus vs. place vs. response. Journal of Comparative \& Physiological Psychology, 48, 19-23.

Wallace, D. G., Hines, D. J., Pellis, S. M., \& Whishaw, I. Q. (2002). Vestibular information is required for dead reckoning in the rat. Journal of Neuroscience, 22, 10009-10017.

Whishaw, I. Q., Hines, D. J., \& Wallace, D. G. (2001). Dead reckoning (path integration) requires the hippocampal formation: Evidence from spontaneous exploration and spatial learning tasks in light (allothetic) and dark (idiothetic) tests. Behavioural Brain Research, 127 49-69.

\section{APPENDIX \\ Calculation of Chance Performance for Alternation in a Plus-Maze}

The rat starts in Arm 1. Because immediate repeats are not counted, it has a 3/3 probability of making a correct response after leaving Arm 1; a correct response is choosing any arm not previously selected, and thus, any response is correct for the first choice. After entering an arm, the rat again has $3 / 3$ probability of making a correct choice; none of those arms were selected previously. Now, on its third choice, only $2 / 3$ arms are correct; it has entered one of those arms previously. On the fourth choice, only $1 / 3$ choices is correct. Scoring is out of five choices; thus, the rat has one more choice, which can be any arm, and the probability of a correct choice is $3 / 3$. Thus, the partial probability is

$$
(3 / 3)(3 / 3)(2 / 3)(1 / 3)(3 / 3)=.222 \text {. }
$$

However, that repetition of an arm that is shown as the last choice above does not have to be made there. It can occur after the second choice or the third choice. Thus,

$$
(3 / 3)(3 / 3)(1 / 3)(2 / 3)(1 / 3)=.074
$$

and

$$
(3 / 3)(3 / 3)(2 / 3)(2 / 3)(1 / 3)=.148 .
$$

Adding these probabilities together results in a probability of .444 , or $44.4 \%$. 